\title{
Recent Trends in the Epidemiology of Liver Abscess in Western Region of Uttar Pradesh: A Retrospective Study
}

Wasif Mohammad Ali", Imad Ali, Rizvi SAA, Rab AZ and Meraj Ahmed

Department of Surgery, Jawaharlal Nehru Medical College and Hospital, AMU, Aligarh, Uttar Pradesh, India

*Corresponding author: Wasif Mohammad Ali, Department of Surgery, Jawaharlal Nehru Medical College and Hospital, AMU, Aligarh, Uttar Pradesh, India, Tel: 9897238280; E-mail: aliwasif110@gmail.com

Received Date: July 31, 2018; Accepted Date: August 01, 2018; Published Date: August 05, 2018

Copyright: @2018 Ali WM, et al. This is an open-access article distributed under the terms of the Creative Commons Attribution License, which permits unrestricted use, distribution, and reproduction in any medium, provided the original author and source are credited.

\begin{abstract}
Liver abscess remains an important clinical entity. It is the most common intra-abdominal visceral abscess. It has become one of the most common communicable diseases with much more prevalence in underdeveloped and developing countries. Low socio economic conditions, unsanitary habits, immuno-compromised state and alcohol abuse are significant risk factors for amoebic liver abscess. A total of eighty-nine patients are included in this study all with confirmed diagnosis of liver abscess. Multiple variables were determined, such as the patient's general data, personal, pathological and non-pathological records, symptomatology, vital signs, laboratory tests, cultures, antibiotic therapy, treatment, and follow-up until discharge. In past few years, it has been seen that there is a slight change in the incidence and appearance of this disease. These changes prompted us to study the patients of liver abscess in this region of northern India.
\end{abstract}

Keywords: Liver; Abscess; Recent trends; Epidemiology; Etiology

\section{Introduction}

Liver abscess is no longer an uncommon problem. It remains an important clinical entity centuries before, since the times of Hippocrates. By definition Liver abscesses are cystic space occupying lesions within liver parenchyma [1]. It is the most common intraabdominal visceral abscess [2]. If early diagnosis and treatment is not instituted it's a lethal condition. It has become one of the most common communicable diseases with much more prevalence in underdeveloped and developing countries. Pyogenic, amoebic and fungal are the three major forms of liver abscess. With the advent of chemo-radiotherapy and more cases of immune compromised patients the incidence of fungal liver abscess has also increased [3]. Pyogenic liver abscess is more common in western world while amoebic liver abscess is more commonly found in third world countries, which are under developed, and more people living under lower socio economic conditions. Commonly known etiologies of pyogenic liver abscess are biliary infection, portal vein seeding, direct extension, hepatic artery seeding, penetrating trauma and cryptogenic cause [4]. Solitary lesions in right lobe of liver are more common than multiple abscess cavities in a single lobe or both lobes involvement. Amoebic liver abscess is the most common extra intestinal form of invasive amoebiasis. Entamoeba histolytica and Entamoeba dispar are the two species of amoeba that infects humans [5-8]. E. dispar remains asymptomatic while $E$. histolytica is the parasite that is responsible for all forms of invasive disease. Amoebic liver abscess transmits through faeco-oral route. Low socio economic conditions, unsanitary habits, immuno-compromised state and alcohol abuse are significant risk factors for amoebic liver abscess. USG remains the main diagnostic modality while contrast CT is $95 \%-100 \%$ sensitive $[9,10]$. Diagnosis of pyogenic liver abscess is confirmed by aspiration and positive culture while for amoebic liver abscess cultures are negative or sterile. Anchovy sauce appearance with odorless nature of aspirated pus is a characteristic feature of amoebic liver abscess, which aids in the diagnosis, while the confirmation is done by serological tests for antiamoebic antibodies [11-13]. Serological tests are not routinely used in our set up due to its high cost. In past few years, it has been seen that there is a slight change in the incidence and appearance of this disease. These changes prompted us to study the patients of liver abscess in this region of northern India.

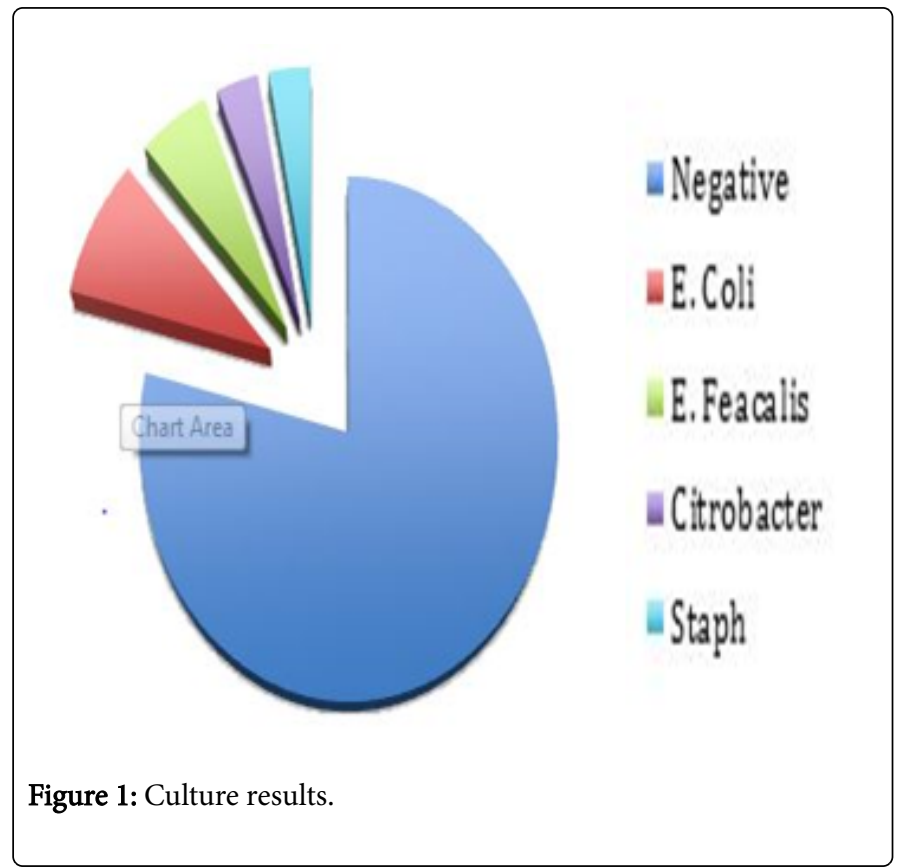

\section{Material and Methods}

A retrospective study was conducted in Department of Surgery at JN Medical College and Hospital, AMU, Aligarh; over a period of 2.5 years from January 2016-May 2018. Eighty-Nine patients with confirmed diagnosis of liver abscess are included in the study and data 
from records were abstracted. Multiple variables were determined such as the patient's general data, personal, pathological and nonpathological records, symptomatology, vital signs, laboratory tests, cultures, antibiotic therapy, treatment, and follow-up until discharge. The investigations performed in all the patients were complete blood counts, kidney and liver function tests, viral markers, abdominal USG, chest and abdominal radiographs, blood cultures, lower GI endoscopy and aspiration study. All the collected data was analysed (Figure 1).

\section{Results}

A total of eighty-nine patients are included in this study all with confirmed diagnosis of liver abscess. All patients were admitted and followed till discharge. Males $(n=85)$ out numbered females $(n=4)$ with a ratio of $85: 4$, showing that liver abscess is male gender entity. The age ranged between 16-78 years with a mean age of $40.1 \pm 12.42$ years. Highest incidence is found in age group of 31-40 years with $n=29$ (32\%). Mean duration of symptoms before presentation in all cases was 8 days. Out of 89 patients fever is the most frequent symptom noted in 66 patients $(74 \%)$ followed by abdominal pain in 42 patients $(47 \%)$. Jaundice, shock, cough, diarrhoea, tender hepatomegaly, malaena, respiratory discomfort are the other symptoms associated with which patients presented, summarized in (Table 1).

\begin{tabular}{|l|l|}
\hline Symptoms & Number \\
\hline Fever & $66(74 \%)$ \\
\hline Abdominal pain & $42(47 \%)$ \\
\hline Jaundice & $17(19 \%)$ \\
\hline Shock & $15(17 \%)$ \\
\hline Vomiting & $14(16 \%)$ \\
\hline Diarrhea & $10(11 \%)$ \\
\hline Acute abdomen & $7(8 \%)$ \\
\hline
\end{tabular}

Table 1: Most common symptoms presented in patients with liver abscesses.

Eight patients (9\%) had diabetes and $5(6 \%)$ were hypertensive. 7 (8\%) patients came out to be Hepatitis +ve while 2 were reactive for HIV (2.2\%), confirmed by ELISA (Table 2). Regarding the imaging studies performed, USG was conducted in all 89 patients. Abscesses were located on the right side in $59(66 \%)$ patients, on the left side in 8 (9\%), and on both sides in 21(24\%). There was 1 abscess reported in 55 (62\%) patients, 2 in $19(21 \%)$, and 3 or more in $15(17 \%)$. The largest reported size was $16 \mathrm{~cm}$, with average size of $7.96 \mathrm{~cm} \pm 4.7 .77(85 \%)$ patients were managed by USG guided needle aspiration while pigtail insertion was done in $12(14 \%)$ patients (Table 3). Of all 89 patients aspirated samples were taken and sent for culture and sensitivity. Results showed a total of $19(21.3 \%)$ positive and 70 (79\%) negative culture results. Of the positives, 8 had E.coli, 5 had Enterococcus faecalis, 3 had Coagulase-negative Staphylococcus and 3 had Citrobacter species. Of the 89 aspirates 63 (71\%) were anchovy sauce in appearance, of which 60 were sterile on culture (Table 4). Out of 89 patients $52(58.4 \%)$ had a history of alcoholism, most of them were habitual drinkers, which points towards a predisposition in such patients. Of the 52 alcoholic patients, 44 were amoebic and 8 were pyogenic liver abscess. 7 (8\%) patients presented with acute abdomen in which emergency exploratory laparotomy was performed. Among the complications that the subjects had encountered during the course were respiratory discomfort, pleural effusion, lower GI bleed, caecal perforation, jaundice, intra abdominal and intra pleural rupture. Lower GI endoscopy was done in all the subjects of whom 7 had positive findings. 3 were having multiple ulcers in colon and caecum, 1 with rectosigmoid ulcer, 1 with proctitis with rectosigmoid ulcer, 1 with internal haemorrhoids and 1 with healed colon ulcers (Table 5). All the patients with pyogenic liver abscess were treated with aspiration+antibiotic therapy sensitive to culture. Standard dose of metronidazole were given to patients of amoebic liver abscess, with aspiration in required cases. All the patients responded well to the treatment.

\begin{tabular}{|l|l|}
\hline Age-group (years) & Frequency $(\mathbf{n})$ \\
\hline $11-20$ & $6(6.7 \%)$ \\
\hline $21-30$ & $22(24.7 \%)$ \\
\hline $31-40$ & $29(32.6 \%)$ \\
\hline $41-50$ & $19(21.3 \%)$ \\
\hline $51-60$ & $10(11.2 \%)$ \\
\hline $61-70$ & $2(2.2 \%)$ \\
\hline $71-80$ & $1(1.1 \%)$ \\
\hline
\end{tabular}

Table 2: Age wise distribution of cases.

\begin{tabular}{|l|l|l|}
\hline & Organism & Numbers \\
\hline 1 & E. Coli & 8 \\
\hline 2 & Enterococcus faecalis & 5 \\
\hline 3 & Citrobacter sp. & 3 \\
\hline 4 & Staphylococcus & 3 \\
\hline
\end{tabular}

Table 3: Showing culture results.

\begin{tabular}{|l|l|}
\hline Right lobe & $59(66 \%)$ \\
\hline Left lobe & $8(9 \%)$ \\
\hline Both lobes & $21(24 \%)$ \\
\hline
\end{tabular}

Table 4: Localisation of liver abscess.

\begin{tabular}{|l|l|}
\hline Abscess number & Subjects \\
\hline One & $55(62 \%)$ \\
\hline Two & $19(21 \%)$ \\
\hline Three or more & $15(17 \%)$ \\
\hline
\end{tabular}

Table 5: No. of abscesses present.

\section{Discussion}

Liver abscess is one of the most important clinical entity confronted by medical practitioners in northern India. The two most common varieties of liver abscess (pyogenic and amoebic) are usually 
indistinguishable clinically and further investigations or invasive procedures are usually needed to establish the diagnosis as they have different lines of treatment $[14,15]$. The presenting symptoms are usually similar with fever and abdominal pain being the most commonly noted. There is male predominance in both pyogenic and amoebic liver abscess; the reason may be more alcohol consumption in this gender with a peak incidence in 31-40 yrs age group.

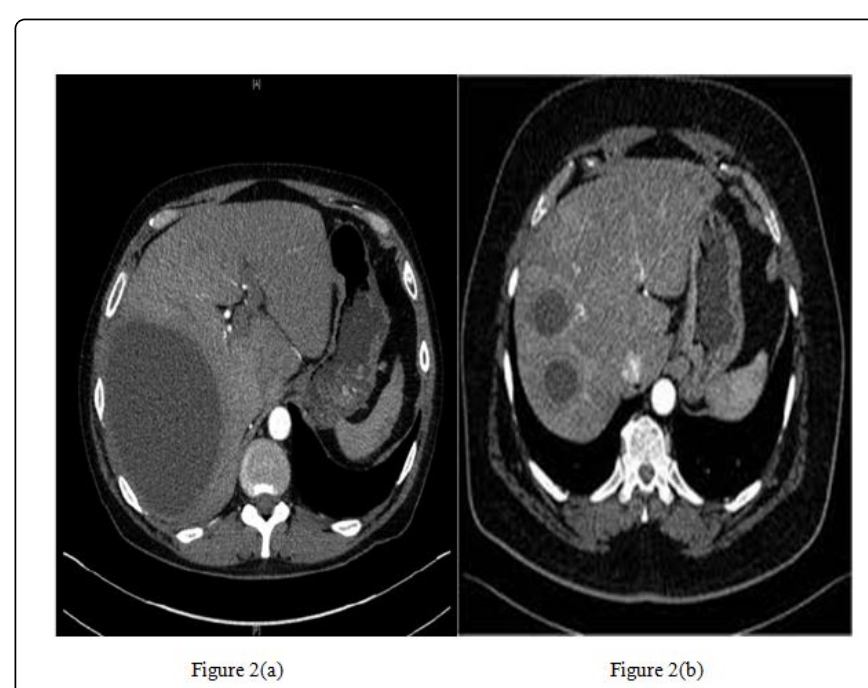

Figure 2: CT scans of liver abscess (a) Single large cavity of abscess in $\mathrm{rt}$ lobe of liver. (b) Multiple cavities.

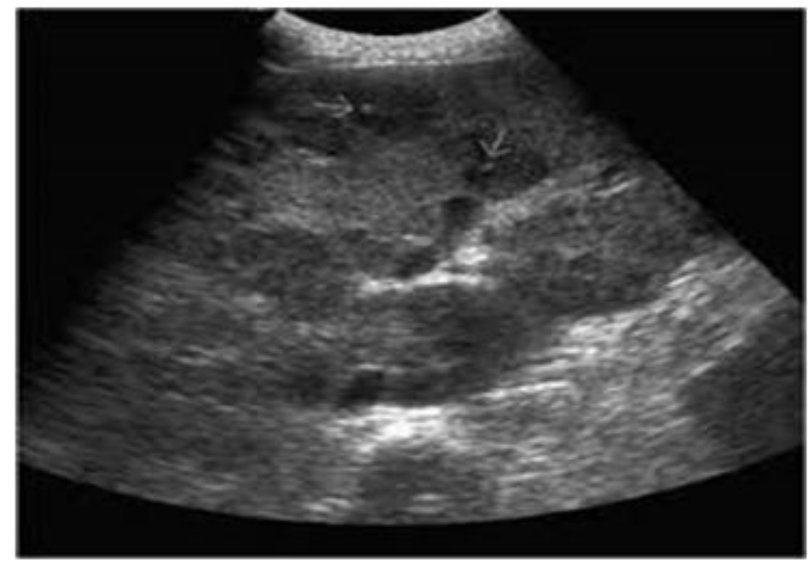

Figure 3: Multiple cavities of liver abscess in ultrasonography.

With the development of new radiologic techniques, improvement in microbiologic identification, advancement of drainage techniques and operative procedures, the mortality rate has come down. Yet the incidence rate has relatively remained unchanged but with the early diagnosis and treatment, the mortality has come down to a significant level in those patients attending to hospitals early. In our study the most commonly isolated organism found is $E$. coli. In our study the incidence of amoebic liver abscess is more than that of pyogenic liver abscess. Fever is the most common symptom noted followed by abdominal pain. Ultrasonography by an experienced radiologist is the first line imaging modality in patients suspected of liver abscesses. CT scan may confirm the ultrasonographic diagnosis, if the ultrasound is normal in case of a high level of clinical suspicion. Ultrasonography is relatively cheaper but the results are totally operator dependent $[16,17]$. It is also possible to aspirate the abscess using USG guidance for diagnostic as well as therapeutic purpose. The distinction between pyogenic and amoebic liver abscess is difficult clinically and even by these imaging studies, and further investigations are needed to differentiate between them. The diagnosis of pyogenic liver abscess was confirmed after culture results while amoebic abscess was confirmed on positive serological results. In our study patients with pyogenic liver abscesses were managed by USG guided aspiration or drain (pigtail) insertion with appropriate antibiotics, as per culture and sensitivity of aspirates. Open surgical drainage was required in patients with acute abdomen resulting due to intraperitoneal rupture of abscess and in caecal perforation. On the other hand, amoebic liver abscesses are treated successfully with amoebicidal drug therapy (Figure 2). Therapeutic aspiration of amoebic liver abscess was done in the following situations: a) high risk of abscess rupture, as defined by cavity size greater than $5 \mathrm{~cm}$; b) left lobe liver abscess, which is associated with higher mortality and frequency of peritoneal leak or rupture into the pericardium; c) failure to observe a clinical medical response to antibiotic therapy within 5-7 days; and d) cannot differentiate from a pyogenic liver abscess. In our series, all patients with amoebic liver abscess underwent ultrasound-guided aspiration and showed good response to metronidazole therapy. However, due to early diagnosis and aggressive management of the condition, as well as of developed complications, no mortality was recorded in this study (Figure 3).

\section{Conclusion}

Liver abscess, both pyogenic and amoebic, continue to be a life threatening condition, with much more prevalence in underdeveloped and developing countries. It is uniformly fatal if left untreated. Male predominance is found with $31-40$ yrs is the most common affected age group. Amoebic abscess has direct relation with alcohol consumption. USG is the investigation of choice as it is cost effective, easily accessible and diagnosis of a liver abscess is possible with reasonable accuracy. Here, in this study we have described the recent trends in the epidemiology of liver abscess at our tertiary care center.

\section{Conflict of Interest}

The authors declare that there are no conflicts of interests regarding the publication of this article.

\section{References}

1. "Liver Abscess Definition in Medical Conditions Dictionary". medconditions.net. 11 April 2018. Retrieved 11 April 2018.

2. Kurland JE, Brann OS (2004) Pyogenic and amebic liver abscesses. Curr Gastroenterol Rep 6: 273-279.

3. Lipsett PA, Huang CJ, Lillemoe KD, Cameron JL, Pitt HA (1997) Fungal hepatic abscesses: Characterization and management. J Gastrointest Surg 1: 78-84.

4. Huang CJ, Pitt HA, Lipsett PA, FA Osterman, KD Lillemoe, et al. (1996) Pyogenic hepatic abscess. Changing trends over 42 years. Ann Surg 223: 600-609.

5. Ellen L, Stanley SL (1996) Protozoa. Amebiasis. Gastroenterol Clin North Am 25: 417-492. 
Citation: Ali WM, Ali I, Rizvi SAA, Rab AZ, Ahmed M (2018) Recent Trends in the Epidemiology of Liver Abscess in Western Region of Uttar Pradesh: A Retrospective Study. J Surg Anesth 2: 117.

Page 4 of 4

6. WHO (1997) WHO/PAHO/UNESCO. Consultation of experts on amoebiasis. Epidemiol Bull 18: 13-14.

7. Aucott JN, Ravdin JI (1993) Amebiasis and "nonpathogenic" intestinal protozoa. Infect Dis Clin North Am 7: 467-485.

8. Kain KC, Boggild AK (2004) Amebiasis. In: Rakel RE, Bope ET, editors. Conn's Current Therapy. 56th edn. Philadelphia: WB Saunders Co pp. 60-63.

9. Johannsen EC, Sifri CD, Madoff LC (2000) Pyogenic liver abscesses. Infect Dis Clin North Am 14: 547-563.

10. Perez JAA, Gonzalez JJ, Baldonedo RF, Sanz L (2001) Clinical course, treatment and multivariate analysis of risk factors for pyogenic liver abscess. Am J Surg 181: 177-186.

11. Knobloch J, Manweiler E (1983) Development and persistence of antibodies to Entamoeba histolytica in patients with amebic liver abscess: Analysis of 216 cases. Am J Trop Med Hyg 32: 727-732.

12. Espinosa-Cantellano M, Martinez-Palomo A (2000) Pathogenesis of intestinal amebiasis: From molecules to disease. Clin Microbiol Rev 13: 318-331.
13. Salit IE, Khairnar K, Gough K, Pillai DR (2009) A possible cluster of sexually transmitted Entamoeba histolytica: Genetic analysis of a highly virulent strain. Clin Infect Dis 49: 346-353.

14. Lodhi S, Sarwari AR, Muzammil M, Salam A, Smego RA (2004) Features distinguishing amoebic from pyogenic liver abscess: A review of 577 adult cases. Trop Med Int Health 9: 718-723.

15. Kimura K, Stoopen M, Reeder MM, Moncada R (1997) Amebiasis: Modern diagnostic imaging with pathological and clinical correlation. Semin Roent 4: 250-275.

16. Elzi L, Laifer G, Sendi P, Ledermann HP, Fluckiger U, et al. (2004) Low sensitivity of ultrasonography for the early diagnosis of amebic liver abscess. Am J Med 117: 519-522.

17. Chavez-Tapia NC, Hernandez-Calleros J, Tellez-Avila FI, Torre A, Uribe M (2009) Image-guided percutaneous procedure plus metronidazole versus metronidazole alone for uncomplicated amoebic liver abscess. The Cochrane Library. 